\title{
Searches for high-mass resonances decaying to pairs of bosons using the ATLAS detector
}

\section{Kirill Grevtsov*, on behalf of the ATLAS collaboration}

Deutsches Elektronen-Synchrotron (DESY), Notkestr. 85, 22607 Hamburg, Germany

E-mail: kirill.grevtsovecern.ch

\begin{abstract}
Several beyond the Standard Model theories predict the existence of new heavy particles decaying into pairs of gauge bosons. This review summarizes the latest ATLAS results on searches for resonances decaying into $W, Z$, Higgs bosons or photons, based on datasets of 36 and $80 \mathrm{fb}^{-1}$ of $p p$ collision data collected at $13 \mathrm{TeV}$. No excess of events is found over the Standard Model background-only expectation, exclusion limits are set for various hypotheses in a broad mass range.
\end{abstract}

The 39th International Conference on High Energy Physics (ICHEP2018)

4-11 July, 2018

Seoul, Korea

${ }^{*}$ Speaker. 


\section{Introduction}

The discovery of a Higgs-like particle announced by the ATLAS and CMS collaborations in $2012[1,2]$ has been an important milestone in the understanding of the mechanism of electroweak (EW) symmetry breaking. The next step is to figure out whether this particle is the only one or if it is part of an extended Higgs sector as predicted by several extensions of the Standard Model (SM): composite Higgs models, heavy vector triplets and models with warped extra dimensions.

In these proceedings a collection of results is presented where the heavy resonances decay into diboson final states. The analyses are separated according to the diboson composition $(W, Z, \gamma)$ and the decays of the bosons. Searches for heavy resonances decaying to $W W$ with leptonic decays of $W$ bosons are described in Section 2 using $36 \mathrm{fb}^{-1}$ of data. A search for fully hadronic decays of $V V(W W, W Z, Z Z)$ is described in Section 3 using $80 \mathrm{fb}^{-1}$ of data, results of a search for a photon and a hadronically decaying boson $(Z, W$ or $H)$ are presented in Section 4 based on a dataset corresponding to an integrated luminosity of $36 \mathrm{fb}^{-1}$. The brief overview of searches are summarised in Section 5.

\section{2. $W W \rightarrow e v \mu v$ decays}

The search for a neutral heavy resonance $H$ decaying to $W W$ with a final state of $e v \mu v$ is described in detail in [3]. The search is performed for the production via quark-antiquark annihilation $(q \bar{q} A)$, vector-boson fusion (VBF) or gluon-gluon fusion $(g g F)$ process covering a mass range from $200 \mathrm{GeV}$ up to $5 \mathrm{TeV}$ for various benchmark models: a Higgs-like scalar in narrow (N) or large (L) width approximation scenarios ${ }^{1}$ a two-Higgs-doublet model (2HDM), the Georgi-Machacek (GM) model, a heavy vector triplet model (HVT), a warped extra dimension model predicting Kaluza-Klein graviton excitations $\left(G_{K K}\right)$ and an effective Lagrangian model (ELM).

The analysis studies opposite sign, different flavour leptons and categorises events into three signal regions (SR), two VBF enriched and one targeting $g g F$ and $q \bar{q} A$. The main backgrounds $(t \bar{t}$ and non-resonant $W W$ ) are estimated with simulations and normalised using dedicated CRs, while the $W+$ jets background was obtained with data-driven techniques.

As no excess over the background prediction is observed, upper limits at $95 \% \mathrm{CL}_{s}$ on $\sigma_{H} \times$ $B(H \rightarrow W W)$ are set for $g g F(\mathrm{VBF})$ in the range of 0.2-4(3) TeV. Values above 6.4(1.3) pb at $m_{H}=200 \mathrm{GeV}$ and above $0.008(0.006) \mathrm{pb}$ at $4(3) \mathrm{TeV}$ are excluded at $95 \% \mathrm{CL}$ by the quasi-inclusive $g g F(\mathrm{VBF})$ NWA analysis. Constraints obtained in the LWA approximation, for all values of $\Gamma_{X} / m_{X}$ are comparable to those obtained in the NWA case. The NWA limits are translated into exclusion contours for Type I and Type II $2 \mathrm{HDM}$ in the plane of $\tan \beta$ versus $\cos (\beta-\alpha)$. The current sensitivity is not sufficient to exclude the GM signal with masses between $200 \mathrm{GeV}$ and $1 \mathrm{TeV}$. Heavy vector triplet signals below about $1.3 \mathrm{TeV}$ are excluded at $95 \% \mathrm{CL}$. The observed limits exclude a KK graviton signal lighter than $1.1 \mathrm{TeV}(750 \mathrm{GeV})$ with the $k / \bar{M}_{P l}=1(0.5)$, while the current sensitivity is not sufficient to exclude the ELM spin-2 VBF signal.

\section{3. $V V \rightarrow q q q q$}

A search for narrow resonances decaying into $W W, W Z$ or $Z Z$ boson pairs, with hadronic decays of $V$, is performed with $80 \mathrm{fb}^{-1}$ as described in [5]. The search covers diboson resonances

\footnotetext{
${ }^{1}$ The LWA approximation is studied for three hypotheses on the ratio between the resonance width and mass: $1 \%$, $5 \%$, and $10 \%$
} 
with masses in the range of 1.2-5.0 TeV for two specific benchmark models: a spin-1 HVT model with signals such as $W^{\prime}$ and $Z^{\prime}$ and a spin-2 Kaluza-Klein graviton.

Bosons produced in the decay of $\mathrm{TeV}$-scale resonances are highly boosted and therefore are reconstructed in ATLAS as a single large radius parameter jet. This analysis uses a new unified object built from both tracking and calorimeter information, referred to as Track-CaloCluster [6]. Jet substructure and mass are exploited to enhance the separation between signal boson jets and jets from multijet background. The efficiency of the boson tagger is determined in a $V+$ jet $C R$, by fitting the jet mass distribution after applying jet substructure cut. After boson-tagging, the data is categorised in five non-exclusive signal regions with different signal efficiencies. The signal model is taken from simulation and background parametrised with functional form. The modelling of the parametric shape tested in a dedicated fit control region in data.

A signal-plus-background fit is performed on the discriminant distribution $m_{J J}$. No significant deviation is found, therefore upper limits on the production cross section times branching ratio to diboson final states for new resonances with masses between 1.2 and $5.0 \mathrm{TeV}$ are set at the $95 \%$ CL, excluding the production of $W W+W Z$ from the HVT model A(B), with $g_{V}=1(3)$ and masses in the range of 1.20-3.40(1.20-4.15) TeV. Production of a $G_{K K}$ in the bulk RS model with $k / \bar{M}_{P l}=1$ is excluded in the range of $1.20-1.90 \mathrm{TeV}$ and $2.1-2.3 \mathrm{TeV}$, at the 95\% CL.

\section{4. $Z / W / H+\gamma$ hadronic decays}

A search for resonance decays to a photon and a $Z, W$ or Higgs boson with subsequent hadronic decay of these bosons is presented and discussed in details in Ref. [7]. Searches for $Z \gamma(W \gamma)$ are carried out in the mass range of 1.0-6.8 TeV for spin- 0 and 2 (spin-1) signals and for $H \gamma$ in the range of 1.0-3.0 TeV for spin-1 signal in the $q \bar{q} A$ channel.

This analysis looks for energetic photons and boosted bosons reconstructed as one large jet. Events are classified into four categories to improve the expected signal sensitivity, presented by different signal efficiency. The separation is based on exploiting $b$-tagging for $Z$ and Higgs to $b \bar{b}$ (BTAG), cuts on jet substructure (D2) and jet mass window (VMASS) to separate hadronically decaying bosons from quark-gluon initiated jets. The signal is modelled by CrystalBall+gaussian, and the background is modelled as a functional form using spurious signal as uncertainty. $\gamma+$ jet and SM $\gamma V$ are taken from simulation and multijet is estimated from data-driven tecnhiques. The jet-photon mass distribution $\left(m_{J \gamma}\right)$ are used as a discriminant variable.

The largest deviation from the SM expectation, corresponding to a significance of $2.7 \sigma$, is found in the $W \gamma$ search at $m_{J \gamma}=2.5 \mathrm{TeV}$. In the absence of any significant excess, limits are set on hadronic $W$ boson decays. The limit varies from about $10 \mathrm{fb}$ at $m_{X}=1.0 \mathrm{TeV}$ to $0.1 \mathrm{fb}$ for $6.6 \mathrm{TeV}$. The limit on $H \gamma$ production is evaluated for resonance masses between 1 and $3 \mathrm{TeV}$ and varies between $10 \mathrm{fb}$ and $4 \mathrm{fb}$ depending on $m_{X}$. The limits on $Z \gamma$ production, vary from about $10 \mathrm{fb}$ to $0.1 \mathrm{fb}$, for $m_{X}$ between 1.0 and $6.8 \mathrm{TeV}$, for spin- 0 and spin- 2 hypothesis.

\section{Summary}

The ATLAS Collaboration has performed a rich search program for additional heavy resonances decaying into two bosons in various final states, testing several benchmark models. The searches presented in this review used datasets collected in $p p$ collisions at $\sqrt{s}=13 \mathrm{TeV}$ corresponding to 36 and $80 \mathrm{fb}^{-1}$. No excess of events over the SM expectation was found in any of the 
final states taken into account. Figure 1 presents a sketch, combining a variety of upper limits set on the collection of diboson channels, covering various spin hypotheses and a broad mass range from $200 \mathrm{GeV}$ up to $6.8 \mathrm{TeV}$.

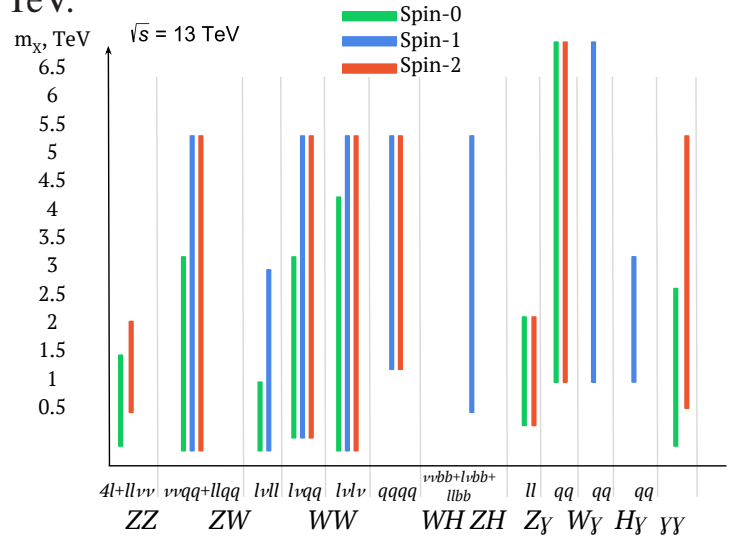

Figure 1: Combining upper limits set on various diboson channels, covering a broad mass range and spin hypotheses - graphical representation of results published in $[3,4,5,7,8,9,10,11]$

\section{References}

[1] ATLAS Collaboration, Observation of a new particle in the search for the Standard Model Higgs boson with the ATLAS detector at the LHC, Phys. Lett. B 716 (2012) 1 [1207. 7214].

[2] CMS Collaboration, Observation of a new boson at a mass of $125 \mathrm{GeV}$ with the CMS experiment at the LHC, Phys. Lett. B 716 (2012) 30 [1207 . 7235].

[3] ATLAS Collaboration, Search for heavy resonances decaying into $W W$ in the ev $\mu v$ final state in $p p$ collisions at $\sqrt{s}=13 \mathrm{TeV}$ with the ATLAS detector, Eur. Phys. J. C 78 (2018) 24 [1710. 01123].

[4] ATLAS Collaboration, Search for heavy ZZ resonances in the $\ell^{+} \ell^{-} \ell^{+} \ell^{-}$and $\ell^{+} \ell^{-} v \bar{v}$ final states using proton-proton collisions at $\sqrt{s}=13 \mathrm{TeV}$ with the ATLAS detector, Eur. Phys. J. C 78 (2018) 293 [1712.06386].

[5] ATLAS Collaboration, Search for diboson resonances in hadronic final states in $79.8 \mathrm{fb}^{-1}$ of pp collisions at $\sqrt{s}=13 \mathrm{TeV}$ with the ATLAS detector, ATLAS-CONF-2018-016 (2018) .

[6] ATLAS Collaboration, Improving jet substructure performance in ATLAS using Track-CaloClusters, ATL-PHYS-PUB-2017-015 (2017) .

[7] ATLAS Collaboration, Search for heavy resonances decaying to a photon and a hadronically decaying $\mathrm{Z} / \mathrm{W} / \mathrm{H}$ boson in pp collisions at $\sqrt{s}=13 \mathrm{TeV}$ with the ATLAS detector, Phys. Rev. D 98 (2018) [1805.01908].

[8] ATLAS Collaboration, Searches for heavy ZZ and ZW resonances in the llqq and $v v q q$ final states in pp collisions at $\sqrt{s}=13 \mathrm{TeV}$ with the ATLAS detector, JHEP 03 (2018) 009 [1708.09638].

[9] ATLAS Collaboration, Search for $W W / W Z$ resonance production in $\ell v q q$ final states in pp collisions at $\sqrt{s}=13 \mathrm{TeV}$ with the ATLAS detector, JHEP 03 (2018) 042 [1710 . 07235].

[10] ATLAS Collaboration, Search for new phenomena in high-mass diphoton final states using $37 \mathrm{fb}^{-1}$ of proton-proton collisions collected at $\sqrt{s}=13 \mathrm{TeV}$ with the ATLAS detector, Phys. Lett. $B 775$ (2017) $105[1707.04147]$.

[11] ATLAS Collaboration, Search for heavy resonances decaying into $a W$ or $Z$ boson and a Higgs boson in final states with leptons and $b$-jets in $36 \mathrm{fb}^{-1}$ of $\sqrt{s}=13 \mathrm{TeV}$ pp collisions with the ATLAS detector, JHEP (2018) 174 [1712.06518]. 